\title{
Three-dimensional seismic velocity structure as determined by double-difference tomography in and around the focal area of the 2005 West off Fukuoka Prefecture earthquake
}

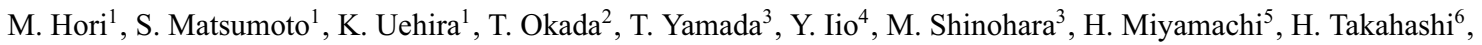 \\ K. Nakahigashi ${ }^{3}$, A. Watanabe ${ }^{1}$, T. Matsushima ${ }^{1}$, N. Matsuwo ${ }^{1}$, T. Kanazawa ${ }^{3}$, and H. Shimizu ${ }^{1}$ \\ ${ }^{1}$ Institute of Seismology and Volcanology, Faculty of Sciences, Kyushu University, Shimabara, Japan \\ ${ }^{2}$ Research Center for Prediction of Earthquakes and Volcanic Eruptions, Graduate School of Science, Tohoku University, Japan \\ ${ }^{3}$ Earthquake Research Institute, University of Tokyo, Japan \\ ${ }^{4}$ Research Center for Earthquake Prediction, Disaster Prevention Research Institute, Kyoto University, Japan \\ ${ }^{5}$ Faculty of Science, Kagoshima University, Kagoshima, Japan \\ ${ }^{6}$ Institute of Seismology and Volcanology, Hokkaido University, Japan
}

(Received November 14, 2005; Revised October 18, 2006; Accepted October 20, 2006; Online published February 2, 2007)

\begin{abstract}
On March 20, 2005 the West off Fukuoka Prefecture earthquake (magnitude of 7.0 on the JMA scale) occurred in southeastern Japan. The earthquake fault was a left-lateral strike-slip having a nearly vertical fault plane and a strike in the WNW-ESE direction. The largest aftershock with a magnitude of 5.8 (JMA) followed 1 month later. To gain more detailed aftershock data, several teams from different Japanese universities jointly installed a number of temporary seismic stations and positioned Ocean Bottom Seismometers (OBSs) immediately above the focal area. Double-difference tomography was used to estimate the three-dimensional (3D) (Zhang and Thurber, 2003) velocity structures in and around the focal area based on the travel time data collected during seismic observations. The high-velocity regions estimated by the inversion are located on the edge of the aftershock area and on the shallow part of asperity, as inferred from the slip distribution. Conversely, the $V p / V s$ ratio is not always as high as that found at the location of the asperity. This finding suggests that the construction of the medium is not uniformly elastic but complex, with different relations between elastic constants and strength.
\end{abstract}

Key words: Double-difference tomography, west off Futuoka prefecture earthquake, 3D velocity structures.

\section{Introduction}

The West off Fukuoka earthquake (magnitude of 7.0 on the JMA scale) occurred on March 20, 2005 in the northern part of Kyushu, Japan. The maximum seismic intensity of 6-lower (JMA scale) was recorded in the Fukuoka and Saga Prefectures. This earthquake killed one person and injured more than 1000 as well as destroying many homes and buildings. Residents of Genkai Island, located directly above the focal area, had to be evacuated due to the massive destruction of houses following the earthquakes and landslides. On the basis of the aftershock distribution and the mechanism inferred from the $P$ wave first motion, the earthquake fault was determined to be a left-lateral strike-slip type with a strike in WNW-ESE direction (Shimizu et al., 2006). The fault strike of the earthquake was almost identical throughout northern Kyushu. One proposed explanation for this strike direction is that the stress field, with the minimum principal axis in the NNW-SSE direction, is loaded onto this region. The principal stress has the same direction as that inferred from the mechanisms of occurrence of microearthquakes in this region (Shimizu et al., 2006).

There is an active fault named Kego passing through

Copyright (c) The Society of Geomagnetism and Earth, Planetary and Space Sciences (SGEPSS); The Seismological Society of Japan; The Volcanological Society of Japan; The Geodetic Society of Japan; The Japanese Society for Planetary Sciences; TERRAPUB
Fukuoka City along the SE extension of the fault, as shown in Fig. 1. The geometrical interrelation between the earthquake fault and the Kego fault suggests that the earthquake fault motion had a positive effect on the stress condition of the Kego fault. An evaluation of the interaction between the two faults requires a study of the velocity structure in this region; however, at the time of the March 20, 2005 earthquake there was not a sufficient number of seismic stations positioned in this region since the seismic activity had been extremely low prior to the earthquake. With the aim of studying aftershock data in more detail, several teams from different Japanese universities installed temporary seismic stations (Shimizu et al., 2006). The stations were positioned on both land and in sea areas. The collective observations from these stations provided a more accurate distribution of the hypocenter (Uehira et al., 2006), particularly in terms of improved accuracy in determining the depth of the hypocenter.

It is important to have a good understanding of the heterogeneous structure of the region when investigating the mechanism of an inland earthquake. In this context, velocity structures in focal areas of large earthquakes provide valuable information on the earthquake rupture process (e.g. Zhao and Negishi, 1996; Shibutani et al., 2005). Velocity structures around focal areas have recently became measurable in detail using a technique called double-difference 


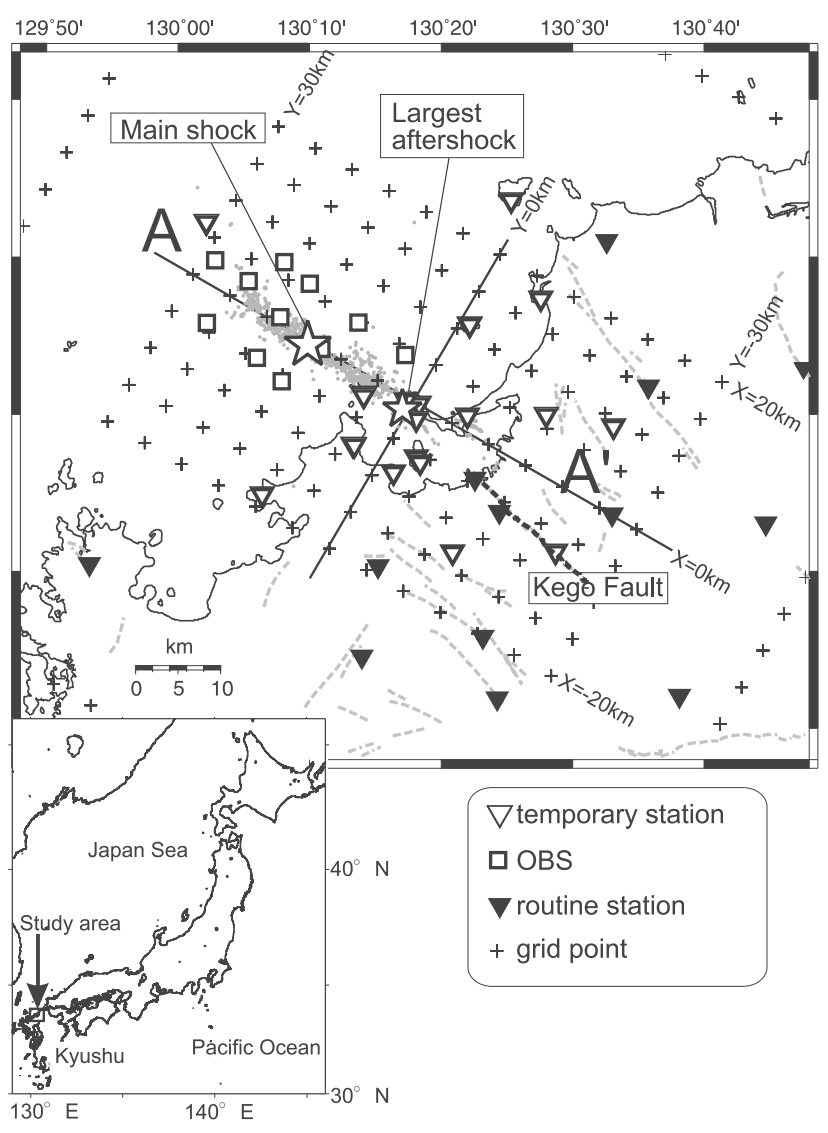

Fig. 1. Location of seismic stations, grids, and earthquakes (dots) used in this study. Broken lines indicate active fault locations.

(DD) tomography, which was developed by Zhang and Thurber (2003). Using this technique, Okada et al. (2004) showed in the case of the 2000 Western Tottori earthquake $(M=7.2)$ that a high-velocity region in the fault corresponding to the large slip area (called asperity) was accompanied by the occurrence of the main shock.

We report here the acquisition of detailed threedimensional (3D) seismic velocity structure data in and around the focal area of the 2005 West-off Fukuoka Prefecture earthquake by means of DD tomography.

\section{Data and Analysis}

In the period following the earthquake, we installed 22 seismic stations around the aftershock area jointly with several universities (Shimizu et al., 2006). These stations were located on land and were equipped with off-line and on-line recording systems. Eleven Ocean Bottom Seismometers were positioned directly above the focal area from March 26, 2005 to April 13, 2005 (Shimizu et al., 2006; Uehira et al., 2006). The seismograms analyzed in the present study are based on the data collected from these tempory stations and from the seismic networks of Kyushu University, the Japan Meteorological Agency (JMA) and Hi-net (NIED).

The DD tomography method developed by Zhang and Thurber (2003) is a travel-time tomography analysis for estimating velocity structure. This method was used in the present study to determine the travel-time differences among nearby pairs of events in addition to the absolute travel times. The DD (hypoDD) method was orig-

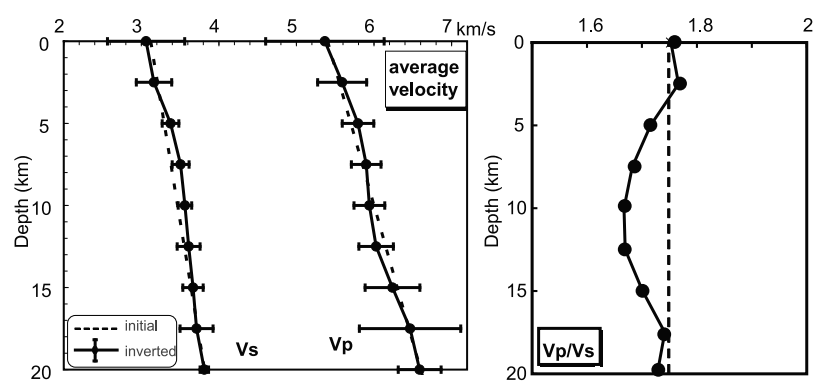

Fig. 2. Depth dependency of average velocity in the reliable region for $P$ - and $S$-waves. Solid lines indicate that the estimated velocity. The dashed lines show the velocity of the initial model. $V p / V s$ ratios for both the final and initial velocity models are shown on the right of the figure.

inally developed by Waldhauser and Ellsworth (2000) as an inversion method for determining a precise hypocenter. Zhang and Thuber (2003) subsequently extended the hypoDD method to tomographic inversion and showed that it has a good resolution in the immediate area around the hypocentral area where the time difference data are directly affected by the velocity structure.

In the present study, data on 953 earthquake events that occurred in the region (latitude: 33.4-34.0 $0^{\circ}$ longitude: 129.75-130.75 ) from March 20, 2005 to May 13, 2005 were provided for the analysis. We used the location of hypocenters routinely determined by the Institute of Seismology and Volcanology (SEVO), Kyushu University, as the initial model for the inversion. Earthquakes that had a hypocenter determined by arrival time data recorded manually at more than ten stations located less than $100 \mathrm{~km}$ from the center focal area were selected. A total of 75 stations were employed in this study. The numbers of arrival time data analyzed are 26,953 for the $P$-wave and 8,908 for the $S$-wave as absolute travel times. To calculate the DD data, we set a maximum hypocentral separation between event pairs of $5 \mathrm{~km}$. The DD analysis included 110,415 $P$-wave data sets and 55,454 $S$-wave data sets. Figure 1 shows the $\mathrm{X}-\mathrm{Y}$ nodes of the velocity model grid located at depths of $0,2.5,5.0,7.5,10.0,12.5,15.0,17.5$, and $20 \mathrm{~km}$, and interval $\mathrm{X}-\mathrm{Y}$ nodes were $5 \mathrm{~km}$ in the central part of the target region $(-20 \leq \mathrm{X} \leq 20 \mathrm{~km}$ and $-30 \leq \mathrm{Y} \leq 30 \mathrm{~km})$. Grid spacing was $10 \mathrm{~km}$ in the remaining parts. The center of the grid distribution was located at latitude $33.68^{\circ}$ and longitude $130.3^{\circ}$. We rotated coordinate system of grid distribution (X-Y) $60^{\circ}$ counterclockwise. Accordingly, the Y-axis is roughly parallel to the strike of the earthquake fault. A one-dimension (1D) seismic velocity model was applied as an initial model for DD tomography. The velocity model is routinely applied to the hypocenter determination in SEVO; it is a depth-dependent model defined as $V(z)=V_{0}+k z$, where $z$ is the depth and $V_{0}$ and $k$ for the $P$-wave are 5.4 and 0.06 , respectively. $V p / V s$ in the initial model is assumed to be 1.73 . The data set is created based on the program 'ph2dt' (Waldhauser, 2001). In order to solve this inverse problem, we used the program "tomoDD1.1" developed by Zhang and Thurber (2003), which takes the station elevation into account and is tunable for weighting factors and other parameters for the iteration scheme. 
a) $2.5 \mathrm{~km}$

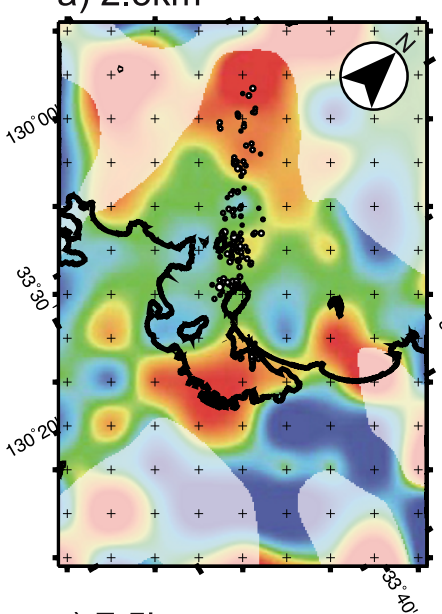

c) $7.5 \mathrm{~km}$

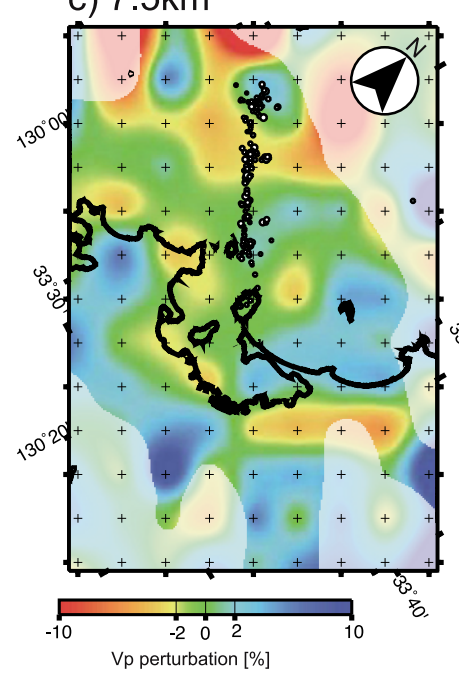

b) $5 \mathrm{~km}$
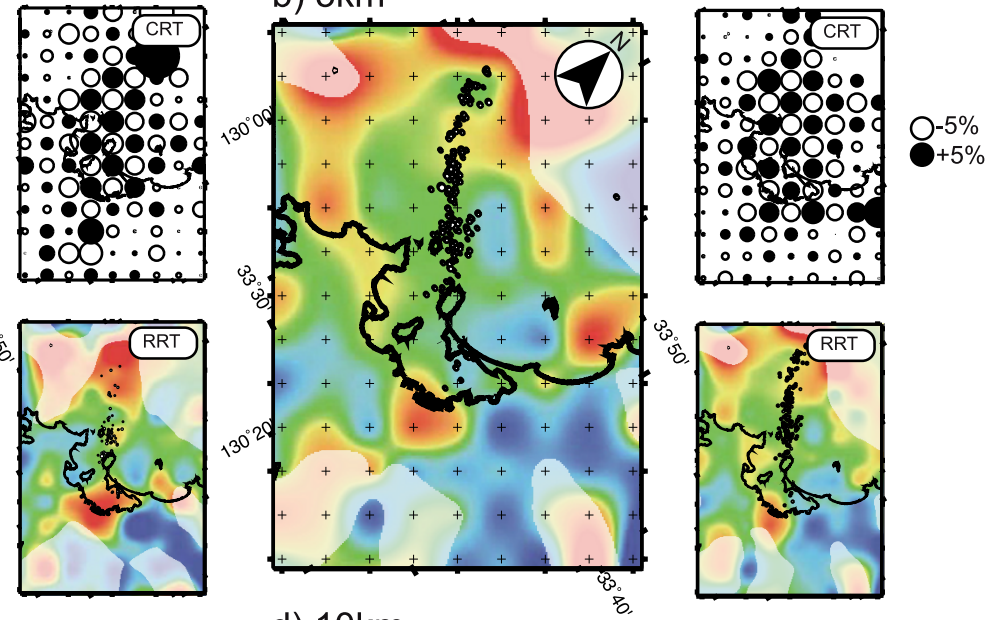

d) $10 \mathrm{~km}$
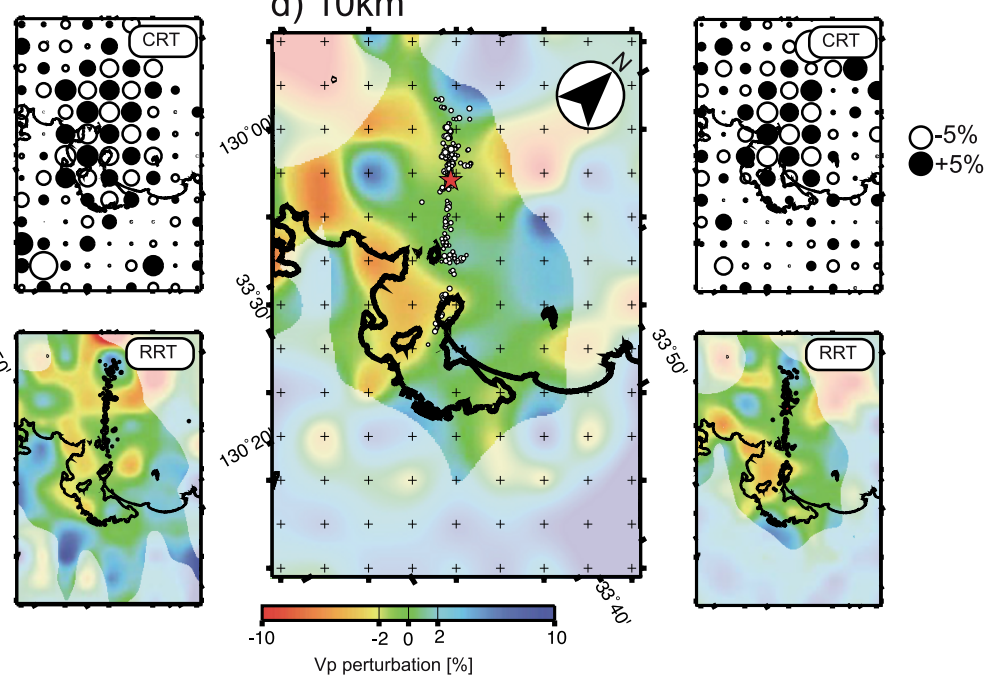

\section{$5 \%$}

Vp perturbation [\%] .

Fig. 3. Horizontal maps of $P$-wave velocity perturbations at depths of $2.5 \mathrm{~km}$ (a), $5.0 \mathrm{~km} \mathrm{(b),} 7.5 \mathrm{~km}(\mathrm{c})$, and $10 \mathrm{~km}$ (d). The area with low reliable solution (i.e., DWS is small) is masked. The RRT results are shown in the upper-right maps. Circles on the left and upper-right maps show the relocated hypocenters within the depth range of $\pm 1.25 \mathrm{~km}$ from the displayed depth. The CRT results are shown in the lower-right maps. The size of the circle in the CRT indicates the proportion of the deviation of velocity from the average value. In (a), the white line indicates the Kego fault.

In order to check the resolution of the tomographic inversion, we performed a checkerboard resolution test (CRT) and a restoring resolution test (RRT). In CRT, we assigned a velocity perturbation of $\pm 5 \%$ to the initial values at grid nodes as a model structure. The perturbation at a grid point was given as an opposite sense to adjacent grid points. We calculated synthetic travel times for all pairs of stations and events for the given model structure. Furthermore, we added Gaussian random noise with a standard deviation of $0.05 \mathrm{~s}$ for $P$-waves and $0.087 \mathrm{~s}$ for $S$-waves to the synthetic travel times as the error in the arrival time data. The velocity structure was then inverted from the test data. Based on the CRT results, we were able to determine that if an inverted value at a grid node has a given velocity, the resolution at the grid point is good. In the RRT, we calculated synthetic travel times based on the structures obtained by inversion from the practical data and added random Gaussian noise in the same way as for CRT. The synthesized data can be used to estimate the velocity structure by the inversion. We compared the obtained structures with the given structures in the two tests, evaluating the spatial resolution of tomography in the target area.

\section{Velocity Structure in and around the 2005 West off Fukuoka Prefecture Earthquake}

The tomographic inversion was carried out according to the scheme described by Zhang and Thurber (2003). As described above, Zhang and Thuber (2003) used both absolute travel-time data and DD data in the program named "tomoDD1.1". The program also takes the altitudes of the stations into account in the calculation of travel time by $3 \mathrm{D}$ ray trace. We performed 16 iterative calculations in the inversion process. The parameters of the inversion-i.e., the number of iterations, weighting factors, and dumping factors-were optimized to maintain stability through the iterative calculations and to give small residuals. For the first half of the iterations, the weighting factors of the absolute arrival time data were tenfold greater than that of DD tomography. The weighting factors of the absolute arrival time data were set to be small (i.e., 0.01 of DD) in the later half of the iterations. The inversion analysis provided root mean square (r.m.s.) residuals of $0.043 \mathrm{~s}$ for the final solution, which were smaller than that of $0.195 \mathrm{~s}$ for the initial model. The r.m.s. residual after the earlier eight iterative calculations was $0.066 \mathrm{~s}$, in which the weighting factor of 

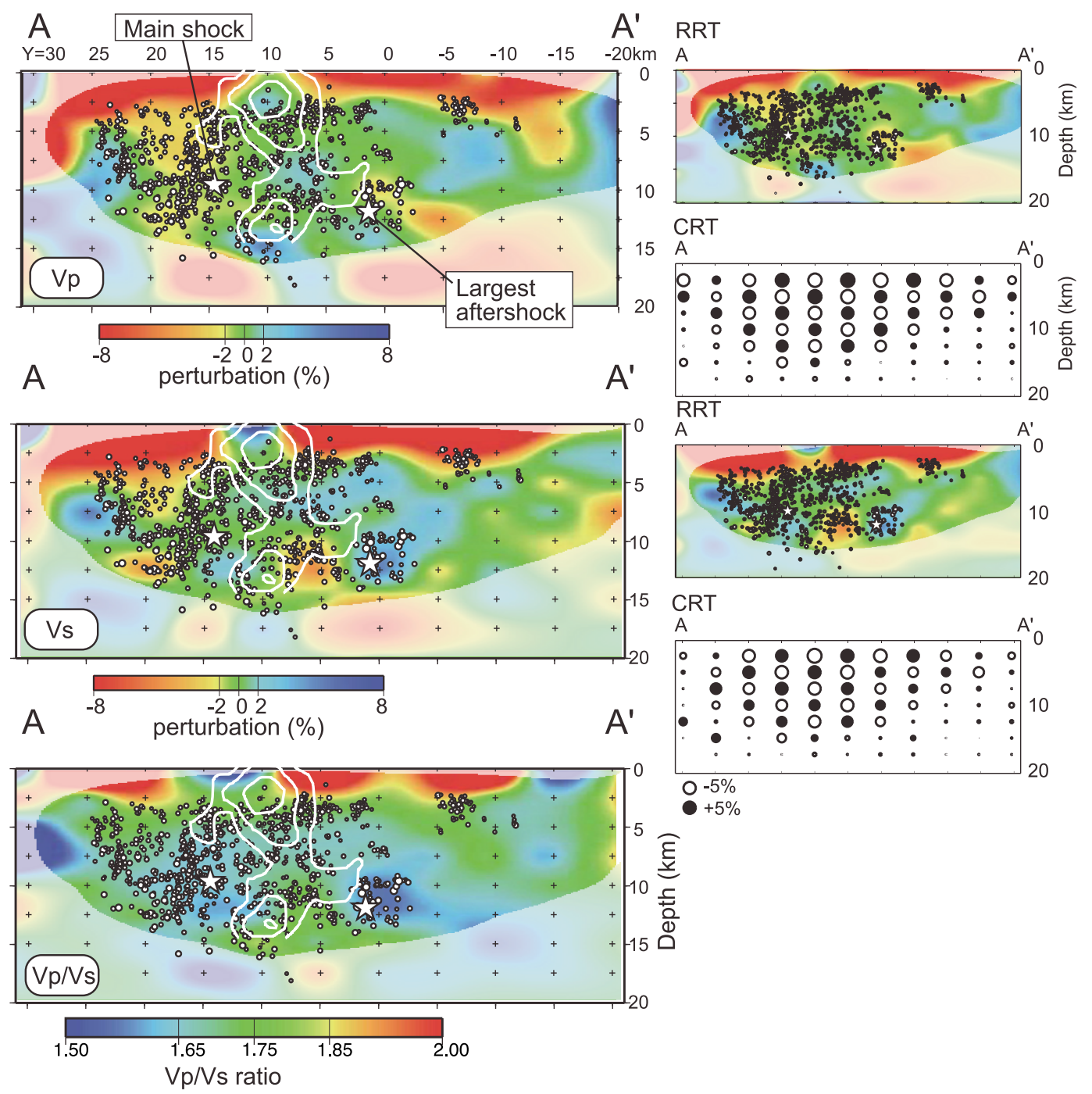

Fig. 4. Vertical cross-sections of $P$ - and $S$-wave velocity and perturbation, and $V p / V s$ ratios along the line A-A' $(\mathrm{X}=0.0 \mathrm{~km})$ in Fig. 1 . White circles and crosses show the hypocenters and grid points, respectively. Stars denote the hypocenters of the main shock and the largest aftershock. White contour lines show the slip distribution at an interval of $0.8 \mathrm{~m}$ (Asano and Iwata, 2005). The results of CRT and RRT for $V p$ and $V s$ are shown in the figures to the right.

the absolute data was large. This result suggested that the final solution was improved by adopting DD data in the inversion.

The velocities of $P$ - and $S$-waves for every depth are shown in Fig. 2. These velocities were calculated by taking the weighted average with DWS as described below. The standard deviations are plotted in the figure as horizontal bars. The depth dependency of the $V p / V s$ ratio is also plotted in Fig. 2. The variation in velocity was almost the same as that of the initial model; however, the velocity near the surface was low relative to the initial value. The $V p / V s$ ratio decreased with increasing depth to $12.5 \mathrm{~km}$; at depths below $12.5 \mathrm{~km}$, the ratio is almost 1.73 . Most of the aftershocks occurred above the depth of $12.5 \mathrm{~km}$, corresponding to increased Poisson's ratio at this depth. However, this feature is not always valid for the whole region, since the well-resolved velocity area in the deeper parts is small in inversion. Figure 3 shows horizontal maps of $P$-wave velocity $(V p)$ perturbations from the average velocity (Fig. 2) at depths of 2.5, 5.0, 7.5 and $10 \mathrm{~km}$. The results of CRT and
RRT are also displayed in this figure. Figure 4 shows the vertical cross-section along the earthquake fault for $V p, V s$ perturbations, and the results of CRT and RRT. The $V p / V s$ ratio is also plotted along the earthquake fault $(\mathrm{X}=0.0 \mathrm{~km})$. The masked areas in these figures are determined with low reliability. The differential weighted sum (DWS) defined by Thurber and Eberhart-Phillips (1999) are small in these areas. The results with DWS $<5 \%$ of the maximum DWS are also masked in the figures. In contrast, the results in the unmasked area in Figs. 3 and 4 are reliable because of the high DWS factor. We confirmed these values in the results drawn from CRT and RRT, as shown in Figs. 3 and 4. The velocity distributions reconstructed by both CRT and RRT are well resolved, even in the unmasked area, implying that the solution obtained is the most reliable one for the aftershock area. This is due to the improved DD tomography in which not only absolute travel time but also DD data are obtainable. All of the results obtained and their stability were checked by running the inversion calculations with different parameters at different grid distributions. In this control 
experiment, the grid distribution was shifted to the $+X$ and $+\mathrm{Y}$ direction with the half-grid interval. For the tests producing r.m.s. residuals similar to that in the original result, the pattern of the velocity perturbation distribution did not change within the unmasked region, indicating that the result in the unmasked area is also reliable.

We investigated the general features of the horizontal distribution of $V p$ perturbations. The $V s$ perturbation shows a pattern similar to that for the $V p$ perturbation. At a depth of $2.5 \mathrm{~km}$, the low $-V p$ region exists in the southeastern part of the target region and corresponds to the area around the Kego fault. The high- $V p$ region is situated just east of the earthquake fault. At depths of 5, 7.5, and $10 \mathrm{~km}$, some regions with either high or low velocity are found around the aftershock area. The high-velocity region exists at the northwestern edge of the aftershock distribution at depths below $5 \mathrm{~km}$. However, we were unable to identify velocity anomalies relating the aftershock distribution. The earthquake fault lies in the area with moderate velocity.

In the vertical cross-section (Fig. 4), the result of CRT and RRT indicate that deeper parts $(\mathrm{Z}>12.5 \mathrm{~km})$ in the focal area provide insufficient resolution. The velocity at the depth of $2.5 \mathrm{~km}$ is low among most of the faults. However, the relatively high- $V p$ and $-V s$ regions exist in shallow parts $(\mathrm{Y}=10 \mathrm{~km}, \mathrm{Z}=2.5 \sim 5.0 \mathrm{~km})$. The high-velocity anomalies are also located at the both edges of the aftershock distribution.

\section{Discussion and Summary}

The low- $V p$ area at the depth of $2.5 \mathrm{~km}$ in the southeastern part corresponds to that around the Kego fault. The high- $V p$ region is found just east of the earthquake fault. The Bouguer gravity anomaly in this region, which was reported by Morijiri et al. (2002) and AIST (2005), shows a similarity to the spatial pattern of velocity perturbation. In the present study, the low-density anomaly corresponds to the low-velocity region, while. the high- $V p$ region corresponds to a high-gravity anomaly. These gravity anomalies are mainly controlled by the thickness of the sedimentary layer. The basement nearby the Kego fault is presumably deeper than that in other areas.

The high- $V p$ regions seen at both edges of the focal area could be interpreted to be a barrier that suppresses the growth of the main shock rupture. Shibutani and Katao (2005) reported that the velocity at the edges of the rupture area was low in the 2000 Western Tottori Earthquake $(M=7.2)$. Their conclusion seemed unusual in that it involved an interpretation of the velocity of the edges that was contradictory to their observations. Their findings suggest that the growth of a rupture in a large earthquake might be suppressed by a medium with either a higher or lower strength (i.e., velocity) than the other part of the fault. In the cross section (Fig. 4), the hypocenters (i.e., rupture initiation points) of the main and the largest aftershocks are located at the edge of the low $V p / V s$ region. Although the strength of a medium is generally reflected in the $V p / V s$ ratio, it is possible that the ruptures started from points at which the strength was relatively weak. However, the lowvelocity region below the hypocenter, as described in other tomographic studies (e.g., Zhao and Negishi, 1996; Okada et al., 2005), was not identified in the present study. Okada et al. (2005) speculated that the existence of a low-velocity area might image the upwelling fluid from the deeper part of the crust. We recognized a low-velocity area at depths below $15 \mathrm{~km}$ corresponding to that reported by others; however, the the resolution was not very high and, therefore, we cannot discuss the relation between the hypocenter and the lower crust in the low-velocity area.

We compared the result of the fault plane (Fig. 4) with the slip distribution of the main shock obtained by the waveform inversion (Asano and Iwata, 2005). The white contours in Fig. 4 indicate the slip distribution accompanying the main shock faulting. The shape of the contour lines suggest that the fault did not slip in the low-velocity region. The large slipping areas correspond to regions with high velocity in shallower part. This is especially striking in terms of the $P$-wave velocity perturbation. At $\mathrm{X}=10$ $\mathrm{km}$, depth=2.5 and $15 \mathrm{~km}$ in Fig. 4, the perturbations of $P$-wave velocity were estimated to be high, showing that the slip became large. Similar results have been obtained in studies of various earthquakes: the 2003 northern Miyagi earthquake (Okada et al., 2005b) and the 2004 mid Niigata Prefecture earthquake (Okada et al., 2005a). In the present study, we found that the large slip area corresponded to the high-velocity region. It is therefore reasonable to consider that a high-velocity medium has a high strength, so that the total slip would become large due to a high stress drop. In such a case, Poisson's ratio (i.e., $V p / V s$ ratio) should become low in the high-strength area. However, this is not always high, as seen in the present study. This finding suggests that the medium is constructed not by a simple uniform elastic medium but by a complex one having different relations between elastic constants and strength. The velocity obtained here is assumed to vary in a much smoother fashion than the grid interval. In order to solve this problem, a higher resolving estimation is needed for the velocity using waveform analyses with short wavelengths, such as scattered wave analysis, that would possibly enable an evaluation of inhomogeneous structures.

Acknowledgments. We are grateful to the teams of Hokkaido University, Tohoku University, University of Tokyo, Kyoto University, Kagoshima University, and Kyushu University for their cooperation. We used the seismic data from JMA, Hi-net (NIED), and Fukuoka city. We also thank two anonymous reviewers for their valuable comments.

\section{References}

Asano, K. and T. Iwata, Source process and near-source ground motions of the 2005 West Off Fukuoka Prefecture earthquake, Earth Planets Space, 58(1), 93-98, 2006.

Geological Survey of Japan, AIST, http://unit.aist.go.jp/igg/rg/ geophysmap-rg/grav/fukuoka_grav_fig.html, 2005.

Morijiri, R., T. Hiroshima, M. Komazawa, M. Makino, Y. Murata, K. Nawa, J. Nishijima and T. Mogi, Bouguer Gravity Anomalies in the Fukuoka area, Kyushu, Japan, Bull. Geol. Surv. Jpn., 53(7/8), 73-594, 2002 (in Japanese with English abstract)

Okada, T., A. Hasegawa, H. Zhang, and C. Thurber, Detailed seismic velocity structure around the focal area of the 2000 Western Tottori earthquake (M7.3) by Double-Difference tomography, 2004 Japan EPS Joint Meeting, J078-P001, 2004.

Okada, T., N. Umino, T. Matsuzawa, J. Nakajima, N. Uchida, T. Nakayama, S. Hirahara, T. Sato, S. Hori, T. Kono, Y. Yabe, K. Ariyoshi, S. Gamage, J. Shimizu, J. Suganomata, S. Kita, S. Yui, M. Arao, S. 
Honda, T. Mizukami, H. Tsushima, T. Yaginuma, Y. Asano, H. Zhang, and $\mathrm{C}$. Thurber, Aftershock distribution and 3D seismic velocity structure in and around the focal area of the 2004 mid Niigata prefecture earthquake obtained by applying double-difference tomography to dense temporary seismic network data, Earth Planets Space, 57, 435440, 2005a.

Okada, T., A. Hasegawa, J. Suganomata, N. Umino, H. Zhang, and C. Thurber, Imaging the fault plane of the 2003 M6.4 northern Miyagi, NE Japan, earthquake by double-difference tomography, Chikyu Monthly, 27(2), 132-138, 2005b (in Japanese).

Shibutani, T., H. Katao, and Group for the dense aftershock observations of the 2000 Western Tottori Earthquake, H, High resolution 3-D velocity structure in the source region of the 2000 Western Tottori Earthquake in southwestern Honshu, Japan using very dense aftershock observations, Earth Planets Space, 57(9), 825-838, 2005.

Shimizu, H., H. Takahashi, T. Okada, T. Kanazawa, Y. Iio, H. Miyamachi, T. Matsushima, M. Ichiyanagi, N. Uchida, T. Iwasaki, H. Katao, K. Goto, S. Matsumoto, N. Hirata, S. Nakao, K. Uehira, M. Shinohara, H. Yakiwara, N. Kame, T. Urabe, N. Matsuwo, T. Yamada, A. Watanabe, K. Nakahigashi, B. Enescu, K. Uchida, S. Hashimoto, S. Hirano, T. Yagi, Y. Kohno, T. Ueno, M. Saito, and M. Hori, Aftershock seismicity and fault structure of the 2005 West Off Fukuoka Prefecture Earthquake ( $\left.M_{\mathrm{JMA}} 7.0\right)$ derived from urgent joint observations, Earth Planets Space,
58, this issue, 1599-1604, 2006.

Uehira, K., T. Yamada, T. Shinohara, K. Nakahigashi, H. Miyamachi, Y. Iio, T. Okada, H. Takahashi, N. Matsuwo, K. Uchida, T. Kanazawa, and H .Shimizu, Precise aftershock distribution of the 2005 West Off Fukuoka Prefecture Earthquake $(\mathrm{Mj}=7.0)$ using a dense onshore and offshore seismic network, Earth Planets Space, 58, this issue, 16051610, 2006.

Waldhauser, hypoDD: a computer program to compute double-difference hypocenter locations, U. S. Geological Survey, Open File Report, 25, $01-113,2001$

Waldhauser and Ellsworth, A double-difference earthquake location algorithm: method and application to the northern Hayward Fault, California, Bull. Seism. Soc. Am., 80, 1548-1368, 2000.

Zhang, H. and C. Thurber, Double-Difference Tomography: the method and its application to the Hayward Fault, California, Bull. Seism. Soc. Am., 93, 1875-1889, 2003.

M. Hori, S. Matsumoto (e-mail: matumoto@sevo.kyushu-u.ac.jp), K. Uehira, T. Okada, T. Yamada, Y. Iio, M. Shinohara, H. Miyamachi, H. Takahashi, K. Nakahigashi, A. Watanabe, T. Matsushima, N. Matsuwo, T. Kanazawa, and H. Shimizu 